\title{
Agrarwende zu See?
}

\section{Die Agrarwende ist in aller Munde. Aber wie sieht es eigentlich mit Lebensmit- teln aus dem Wasser aus, mit Fisch und Meeresfrüchten? Derzeit sind erste Be- mühungen um ein Ökosiegel auch für Fische aus Aquakulturen zu beobachten. \\ Bei der Hochseefischerei ruhen Hoffnungen auf den Aktivitäten des Marine Ste- wardship Council. Schützenhilfe könnte eventuell von den aktuellen WTO-Ver- handlungen kommen.}

$\mathrm{N}$ Von Dierk Jensen und Carmen Becker ichts ist globaler als Fisch. Er wird überall verzehrt, überall gefangen. Ob nun von dänischen Kuttern, russischen Trawlern oder indischen Netzfischern. Und der Hunger nach den schmackhaften Eiweißlieferanten wächst weiter. Zwar nimmt die Fischzucht ständig zu und vermag einen Teil des steigenden Bedarfs abzudecken, doch geht das Fanggeschäft in manchen Hochseegebieten gnadenlos weiter. Bestände werden massiv überfischt, einige Fischarten sind vom Aussterben bedroht.

Diese Gefahr ist bekannt. Sie wird nicht nur von Umweltgruppen heftig kritisiert, sondern auch von Teilen der Fischwirtschaft selber. Auch die FAO, die Ernährungs- und Landwirtschaftsorganisation der Vereinten Nationen, hält seit Jahren ihre Mitgliedsländer an, kriminelle und illegale Fangpraktiken zu bekämpfen. Dabei sind Fangquoten alleine kein wirksames Instrument für eine nachhaltige Fischerei. Daher stand im Februar auch eine FAO-Fachtagung zum Fischhandel in Bremen im Zeichen der Nachhaltigkeit.

Eine Antwort auf die Gefahr der Überfischung von der Angebotseite ist die Zucht. Diese trägt aber nicht immer zur Freude der Verbraucher bei, wie diverse Skandale um Antibiotika und andere wachstumsfördernde Medikamente im Fischfutter zeigen. Eine Alternative zum Zuchtfisch bieten neue Arten, die für den Fischhandel erschlossen werden können. So zum Beispiel der Kingklip aus Südafrika, der dem bedrohten Kabeljau ähnelt, und der Paiche aus den peruanischen Amazonasgebiet.

Letztlich geht es jedoch - ähnlich der vieldiskutierten Agrarwende zu Lande - um effektive Instrumente, die als Pendant zur ökologischen Landwirtschaft eine bestandschonende oder sogar ökologische Fischerei ermöglichen. Wird es also in Zukunft auch eine Art Biosiegel für Fisch und Meeresfrüchte geben?
Für die Hochseefischerei, den „Wildcatch“, sicherlich nicht, wird doch kein Fisch auf Hochsee mit Knochenmehl gemästet. Dagegen macht ein Siegel für Fische aus Aquakulturen Sinn. Der Öko-Anbauverband Naturland ist darin in Deutschland wegweisend und zählt vier Forellenund Karpfenzïchter, die nach Öko-Richtlinien Fische halten. Allerdings darf das deutsche Biosiegel bislang auf keinem dieser Fische „kleben“, weil die EU-Ökoverordnung bis dato die Fischzucht ausklammert. Ein Manko, das Verbraucherministerin Renate Künast im Zuge der Agrarwende beheben möchte. Sie findet dabei beim EU-Agrarkommissar Franz Fischler Gehör. Er will noch in diesem Jahr eine überarbeitete Fassung der Ökoverordnung vorlegen.

\section{Vorreiter Unilever}

Unabhängig davon hat der Unilever-Konzern seine langfristige Geschäftspolitik hinsichtlich der Hochseefischerei umgestellt. „Wir haben uns verpflichtet, bis spätestens 2005 den gesamten Fischeinkauf aus bestandschonender Fischerei zu beziehen", hebt Dierk Peters, Marketing Manager von Unilever/Iglo, hervor. Damit übt der Konzern als einer der weltweit größten Fischaufkäufer großen Druck auf die Branche aus. Beispielsweise bezieht der Konzern seit einiger Zeit kein Fischöl mehr, das mittels Gammelfischerei gewonnen wird. Unilever setzt auf das von ihm selbst mit initiierte Marine Stewardship Council (MSC). Dieser 1996 gegründete Weltmeeresrat hat als unabhängige, nichtstaatliche Organisation einen Regelkatalog für umweltgerechte Hochseefischerei aufgestellt. Wer nach diesen Regeln fischt, kann sich von MSC zertifizieren lassen (1).

Unilever selbst hat bereits Artikel mit MSC-Logo im Sortiment. Zum Beispiel das Schlemmerfilet Champignon. Allerdings mache dieses Segment bisher nur ein paar Prozent des Gesamtangebots aus. „Das Wichtigste im bevorstehenden Umstellungsprozess ist, die Interessen von Verbrauchern und Fischerei miteinander zu verbinden", sagt Peters. Seiner Ansicht nach stehen sich sensibilisierte Verbraucherinteressen und Nachhaltigkeit nicht unüberbrückbar gegenüber.

Das ist im Sinne von Heike Vesper, Fischereireferentin der Umweltorganisation World Wide Fund for Nature (WWF), die zusammen mit Unilever das MSC auf den Weg brachte. Vesper kritisiert allerdings das Tempo des Umdenkens: ,Nach wie vor ist die europäische Fangquote viel höher als wissenschaftlich eigentlich zu akzeptieren ist." Auch machen ihr die Fangtechniken große Sorgen, die den Bestand an Schweinswalen in Nord- und Ostsee ernsthaft bedrohen. Und trotzdem: Sie sieht erste, „wenngleich kleine" Schritte hin zu einer umweltverträglicheren Fischerei. Auch für Greenpeace gehen die Kriterien des MSC in die richtige Richtung, sie würden aber nicht ausreichen. Die Organisation fordert eine Bewirtschaftung der Fischbestände nach dem Vorsorgeprinzip: Beschränkungen und Kontrollen sollen greifen, bevor die Bestände hoffnungslos überfischt sind.

Unerwartete Schützenhilfe könnte dabei von der Welthandelsorganisation WTO kommen. Dort steht das Thema Subventionen und Fischfang auf der Agenda, unter anderem beim Märztreffen des Komitees für Umwelt in Genf. Viele der Fischfangflotten vornehmlich aus industrialisierten Ländern, welche die Fischgründe übermäßig belasten, erhalten von ihren jeweiligen Regierungen Subventionen. Im Zuge der weiteren Liberalisierung des Agrar- und Fischereisektors können Entwicklungsländer kaum noch mithalten. Wie genau die Subventionen im Zusammenhang mit Überfischung stehen, wird an verschiedenen Beispielen derzeit noch analysiert.

\section{Anmerkung}

(1) Vgl. hierzu auch Maier, S.: Strategische Kooperationen zur Sicherung von Zukunftsmörkten. Das Beispiel Marine Stewardship Council. In: Ökologisches Wirtschaften, Nr. $4 / 1999$, S. 9.

\section{Die Autorlnnen}

Dierk Jensen ist Journalist, Carmen Becker ist Mitarbeiterin von Ökologisches Wirtschaften. Kontakt: agenda. fotografen und journalisten, Rothestr. 66, 22765 Hamburg., Tel. 040/ 391907-12, Fax 040/ 393746, E-mail info@agenda-fototext.de (Dierk Jensen); carmen.becker@ioew.de 
(c) 20I0 Authors; licensee IÖW and oekom verlag. This is an article distributed under the terms of the Creative Commons Attribution Non-Commercial No Derivates License (http://creativecommons.org/licenses/by-nc-nd/3.o/), which permits unrestricted use, distribution, and reproduction in any medium, provided the original work is properly cited. 